\title{
Processing of information about location in brief visual displays
}

\author{
DOUGLAS G. LOWE \\ Trent University, Peterborough, Ontario, Canada K9J $7 B 8$
}

\begin{abstract}
Within the context of the selective masking effect (Merikle, Coltheart, \& Lowe, 1971), a series of five experiments investigated the utility of bar-marker probes as a poststimulus sampling procedure for partial report of brief visual displays. It was demonstrated that bar markers are inadequate as partial-report cues because subjects cannot properly locate the positions of the probes. An improved bar-marker procedure was developed which removed this problem of cue localization. Furthermore, it was shown that subjects, when given the opportunity, are able to report much more from brief visual displays than the bar-marker procedure typically permits. It was also suggested that subjects' ability to employ bar-marker probes is governed by quite different factors than their capacity to report the contents of brief visual displays.
\end{abstract}

Recently, it has been reported that backward pattern masking of tachistoscopically presented letter rows produces a selective or differential effect upon accuracy of report (Merikle \& Coltheart, 1972; Merikle, Coltheart, \& Lowe, 1971). Compared to control conditions where presentation of the letter rows is followed by a white field, the presentation of a masking stimulus greatly impairs recall performance at the center positions of the letter rows, but has minimal effect upon performance at either end of the displays.

However, in several unpublished experiments by the present author, it was found that a masking stimulus impaired performance equally at all positions ot the letter rows when recall of single letters was cued by bar markers. That is, relative to no-masking control conditions, the mask disrupted performance equally at all positions of the arrays when both the bar markers and the masking stimulus were presented immediately at display offset.

Merikle et al. interpret the selective masking effect as indicating that the ends of multiletter arrays are processed to a nonmaskable level prior to the center items, and thus, by the time the mask arrives, only the center items are disrupted. In addition, Coltheart (1972) has : : ggested that this "ends-tirst" processing sequence reflects the order in which information is transterred from iconic memory (Neisser, 1967) to a more permanent memory storage system.

This paper is based on part of a dissertation submitted to the University of Waterloo in purtial fultillment of the requirements for the $\mathrm{PhD}$ degree. The author expresses his gratitude to Philip Merikle and Max Coltheart for their advice and support. This research was supported by grants from the National Research Council of Canada to P. M. Merikle (APA-231) and from Trent University of D. G. Lowe. Jan Reitz and Lou ise Brown deserve thanks for collection and tabulation of the data from Experiments $I I I$ and $V$, respectively. Requests for reprints should be sent to Douglas $G$. Lowe, Department of Psychology, Trent University, Peterborough, Ontario, Canada K9J 7B8.
In light of the above interpretations attached to selective masking effects, occurrence of different types of masking effects on letter rows may have considerable importance for an understanding of visual information processing. Subsequently, the present studies were designed to explore further the previous findings that multiletter arrays are masked equally at all positions when recall of single letters is cucd by bar markers. Furthermore, considering the popularity of bar-marker probes as a poststimulus sampling procedure (e.g., Averbach \& Coriell, 1961; Haber, 1969), it seems imperative to investigate the nature of this partial-report (PR) technique, since it apparently yields quite unique masking effects on the letter rows.

\section{EXPERIMENT I}

This study was designed to provide further support for the finding that the presentation of a patterned masking stimulus impairs recall performance equally at all positions of letter rows when recall of single letters is cued by bar markers.

\section{Method}

Design and Materials. All subjects were presented a set of 105 sequences of seven random letters. The set of sequences was constructed so that all letters except $I, O, Q, W$, and $X$ were used five times in each of the seven positions and no letter appeared more than once in a single sequence. The letter sequences were prepared for presentation on white cards in black, uppercase, 18-point letters (Letraset No. 287), and the visual angle subtended by a single sequence was approximately $2.84^{\circ}$ x $0.24^{\circ}$.

For hall the subjects (mask condition), presentation of the letter sequences was followed immediately by a presentation of a visual noise stiniulus. For the remaining subjects, a white field followed presentation of the letters. The visual nolse stimulus, subtending a visual angle of approximately $4.71^{\circ} \times 0.30^{\circ}$, consisted of a rectangular strip containing a pattern of randomly arranged small black and white squares (Gregory, 1966, p. 59). The masking stimulus was arranged so that it completely covered the positions occupied by the letters. 
Recall was cued by bar markers. The bar markers, subtending a visual angle of approximately $0.12^{\circ} \times 0.60^{\circ}$. appeared both above and below a location on the masking stimulus such that the near ends of the bars were approximately $0.44^{\circ}$ distant from the center of the position of the target letters. The bar markers for the no-masking condition were positioned in a similar fashion except that no visual noise stimulus was present.

The order in which the 105 sequences were presented was determined randomly and was identical for each subject, but the position probed on each trial was counterbalanced so that across a block of seven subjects each letter in each position of the 105 sequences was tested once. For each subject, the seven positions were probed equally otten, and the order in which the probes were adninistered was ratidom within the restriction that each position was probed once within each block of seven trials.

Procedure. All materials were presented by means of a Scientific Prototype three-field tachistoscope (Model GB). The luminance of all fields was approxinately $20 \mathrm{fL}$. On each trial, the subjects first viewed a preexposure field containing a small fixation point. Following a verbal ready signal, the subjects initiated a 100 -msec expisure of a letter sequence. Immediately following presentation of a sequence, the bar markers and the noise stimulus (when appropriate) were presented for $50 \mathrm{msec}$. The tachistoscope returned to the preexposure field following each trial. The subjects were required to report the letter indicated by the bar marker and were instructed to guess if they were uncertain of the identity of the cued letter.

Subjects. The seven subjects in each group were male and female students whose participation fulfilled a course requirement at the University of Waterloo. They were assigned to masking and no-masking conditions in an alternating sequence when they appeared at the laboratory for the experiment.

\section{Results and Discussion}

Performance scores for each subject were determined by summing recall scores over the 15 sequences probed for each position in the display. These scores were analyzed by a 2 by 7 analysis of variance to evaluate masking and position. ${ }^{1}$ The results are presented in Figure 1 in terms of mean percent correct identifications at each position in the stimulus display.

As shown in Figure 1, the presentation of the

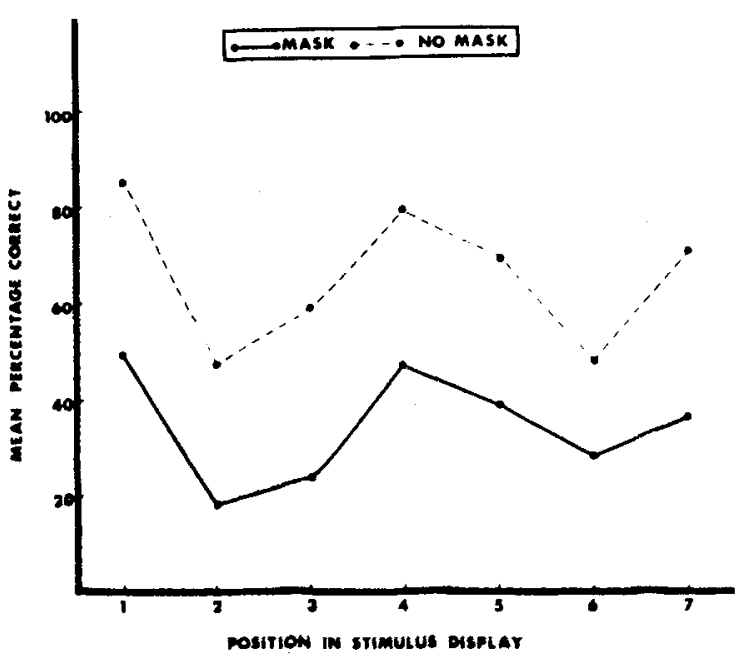

Figure 1. Mean percentage letter recall scores in Experiment I. masking stimulus produced large decrements in performance relative to the no-masking condition, $\mathrm{F}(1,12)=12.92$. $\mathrm{MSe}=39.27$. Overall recall accuracy was $65 \%$ and $34 \%$ for the no-masking and masking groups, respectively. It is also evident from Figure 1 that recall performance varied as a function of position within the letter rows, $F(6,72)=5.45$, $\mathrm{MSe}=9.48$. Accuracy of report across position in the letter rows showed the same general "W-shape" curve reported in other PR studies (e.g., Averbach \& Coriell. 1961; Haber \& Standing, 1969; Merikle et al., 1971). The data in Figure 1 also indicate that performance was impaired equally at all positions of the letter rows by the presentation of the masking stimulus. The analysis of variance provided no evidence that the masking stimulus had a differential effect across the positions of the letter rows, $\mathrm{F}(6,72)<1, \mathrm{MSe}=9.48$.

The results of the present experiment establish that a patterned masking stimulus impairs recall performance equally at all positions of the stinulus arrays when recall is cued by bar markers. Thus, in contrast to the results of Merikle et al. (1971), the use of bar markers as a PR procedure yields quite distinct masking effects at all positions of letter row's.

One possible explanation of these discrepant findings is that the two sets of experiments employed different recall procedures, and subjects may have been differentially successful at utilizing the recall cues. Specitically, in the Merikle et al. experiments, subjects were required to report only the identities (i.e., the names) of the elements in the arrays, while in the present experiment the bar-marker probes necessitated report of the letters on the basis of their spatial locations. Townsend (1973) has recently reported that the task of both locating and identifying the contents of multielement arrays is more difficult than solely reporting the identities of the elements, because information about the locations and identities of the items may not be available to subjects in iconic memory. Therefore, because of a lack of the necessary information. the subjects in the present experiment may hatve encountered extreme difficulty in employing the bar-matker probes.

Furthermore, since a masking stimulus was employed in the present research, the presentation of the mask may have accentuated the problem of cue employment for subjects in the masking condition. As Townsend (1973) has pointed out, in order to utilize the bar-marker probe, subjects must have information about (a) the location of the cued letter relative to the letter display. (b) the location of the bar marker relative to the bar-marker display, and (c) the position of the bar-marker display relative to the letter display, and it is possible that all such types of information would not be available following mask presentation. Subsequently, mask presentation may increase the problems of cue localization and leave 
subjects in the predicament of trying to decide "what was where?" perhaps in itsell a more difficult decision than attempting to identify a degraded tetter signal. Therefore. to the extent that recall performance may be determined more by subjects' ability' to successfully utilize the PR probes than by the amount of material actually processed, selective masking effects may not be evident with the bar-narker procedure because of subjects' interior ability to employ the bar-marker probes.

\section{EXPERIMENTS II AND III}

Experiments $I I$ and III represent attempts to obtain evidence for the previous notions about the problems of mislocalization of the PR probes and concurrently to develop a report procedure which would eliminate these problems. In both experiments, subjects were not required to report letters on the basis or their locations. Instead, on each trial, the same, perfectly predictable, stimulus sequence was presented and subjects were queried only about the positions designated by the probes. In Experiment III, subjects were provided with the combined information of the location of the bar-marker probe relative to the bar-marker display and the location of the bar-marker display relative to the stimulus display, whereas in Experiment II this information was not available.

The rationale of presenting a predictable stimulus array was to remove the uncertainty about the nature of the stimulus array and the relative locations of the elements within the array. In other words, since subjects knew what the elements of the stimulus array were, and especially where they were, the demands of processing the stimulus array are much reduced. Therefore, it is possible to deternine the influence of the absence (Experiment 11) or presence (Experiment III) of the combined information about the positions of the bar markers relative to the bar-marker display and the position of the bar-marker display relative to the stimulus display on subjects' ability to locate the probes. In short. since consideration of the identity of the stimulus array was unnecessary, the task is one of almost pure location pertormance.

\section{Method}

Design and Materials. All subjects were presented the same sequence of seven digits numbered 1 through 7 from left to right. This stimulus sequence was prepared in the same manner as the letter rows used in Experiment I. The masking stimulus was identical to that employed in Experiment I except that, in order to control for any intluence that the mask may have exerted on subjects' judgments about location in Experiment I (e.g.. judging the positions of the bar markers relative to the ends of the mask), the mask was extended across the entire visual field so that neither end was visible.

In both experiments, bar markers were placed upon the masking stimulus. In Experiment II, placement of the bar markers was identical to that of Experiment I, while in Experiment III all positions of the stimulus array were bar-marked. The bur markers were of two types: a single long bar which subtended a visual angle of approximately $0.12^{\circ} \times 0.60^{\circ}$ and six shorter bars, all subtending visual angles of approximately $0.12^{\circ} \times 0.30^{\circ}$.

In both experiments. the same digit sequence was presented on all trials and the order in which the positions were tested was randonized in the same manner as in Experiment $I$.

Procedure. In both experiments, the procedure was identical to that followed in Experiment I. except that subjects were instructed to repon the digit cued by the bar marker. In Experimint III, subjects were instructed to report the digit cued by the longer bar. They were told that the shorter bars were presented to aid them in determining exactly which position was probed. In Experiment II. all subjects were given 315 trials, while each subject in Experiment 111 performed 210 trials.

Subjects. All subjects were male and female undergraduates at the University of Waterloo. The tive subjects in Experiment II were paid $\$ 2$ for their participation, while the participation of the six subjects in Experiment Ill fultilled a course requirement.

\section{Results and Discussion}

Performance scores for each subject were determined by summing the number of correct localizations at each position. A maximum score of 45 and 30 correct localizations at each position was possible in Experiments II and III, respectively. These scores, from each experiment, were submitted to separate repeated-measures analyses of variance to evaluate the effects of position. The results of both experiments are presented in Figure 2 in terms of mean percent correct localizations at each position.

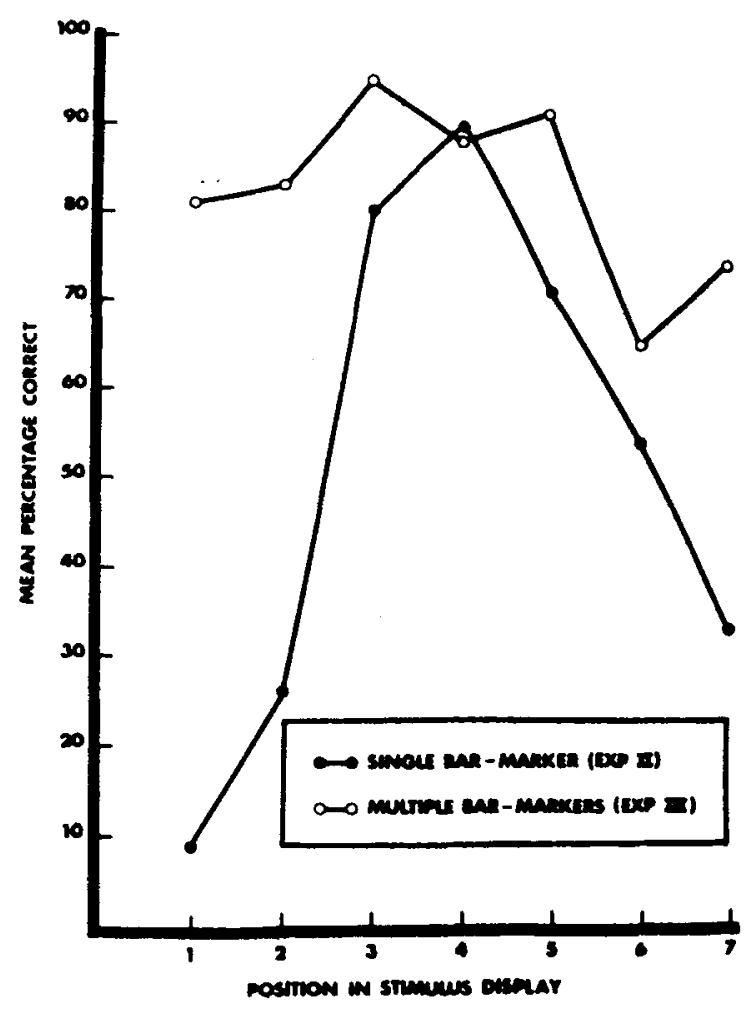

Figure 2. Mean percentage probe localization scores in Experiments II and III. 
As shown in Figure 2, there were large differences in performance in Experiment 11 as a lunction of position probed, $F(6,24)=32.14, \mathrm{MSe}=28.61$. Overall, the percentage correct localizations were $51 \%$, and accuracy ranged from a high of $90 \%$ correct at Position 4 to a low of $9 \%$ correct at Position 1. The data in Figure 2 also indicate that errors in localization increased directly with the distance of the positions from fixation. This inverted "U-shape" function is supported by a significant quadratic trend, $F(1,4)=115.1, \mathrm{MSe}=37.80$.

The results of Experiment II support the suggestion that subjects in Experiment I were experiencing great difficulty in locating the bar markers. It is also clear that difficulty of localization is much more pronounced at the ends of the arrays than at the middle positions. Thus it appears that subjects encounter extreme difticulty in employing the bar-marker cues when they have no information about the location of the probe rei ive to the bar-marker display or to the stinfulus display. Therefore, the reason for the equal masking effects at all positions of the letter arrays in Experiment 1 may have been the subjects' inability to discern exactly which location was being cued, especially under conditions of backward masking. However, since Experiment II did not include a no-masking control condition, it is impossible to assess the influence of localization difficulty on the performance of the no-masking group of Experimcat I. This problem will be dealt with in Experiment V. Nevertheless, the implication of Experiment $1 \mathrm{I}$ is that subjects' inability to correctly locate the probes under backward masking conditions would exert a large influence on recall performance.

Considering the results of Experiment III, the data in Figure 2 also indicate that there were some differences in performance as a function of position probed. The overall correct localizations were $82 \%$, and performance ranged from a high of $95 \%$ correct at Position 3 to a low of $65 \%$ at Position 6. In general, accuracy tended to decrease slightly with distance from fixation; however, the analysis of variance revealed that these differences were not statistically reliable, $F(6,30)=1.44, \mathrm{MSe}=40.07$.

The results of Experiment III indicate that the multiple bar-marker procedure eliminates, for the most part, the cue location problems encountered in Experiment II. Overall performance was much higher in Experiment III than in Experiment II, and, although performance tended to decrease slightly with increases in foveal eccentricity, it is obvious that accuracy at the terminal positions of the row was superior to that obtained with the single bar markers employed in Experiment 1I. Thus, the information provided by the smaller bars about both the location of the probe relative to the bar-marker display and the location of the bar-marker display relative to the stimulus display liminates the difficulties encountered with the single bar marker and permits adequate localization of the probes.

\section{EXPERIMENT IV}

The multiple bar-marker technique appears to be a superior procedure for investigating the processing of brief visual displays; since it may be assumed that recall perfornance will be determined principally by the amount of material actually processed rather than subjects' differential ability to employ the PR cues at different positions of the arrays. Accordingly, Experiment IV was conducted to assess the effect of the multiple bar-marker procedure on recall of single letters. In particular, the experiment was designed to determinc whether the multiple bar-marker procedure would yield a selective masking effect similar to that obtained in previous experiments (e.g., Merikle \&Coltheart, 1971; Merikle et al. 1971). While it appears that the multiple bar-marker procedure eliminates problems of cue localization encountered with a single probe, the utility of the multiple-bar technique for report of the elements of letter arrays is unknown. It is possible that the multiple-bar procedure may not be an aid to letter recall and that the equal masking effects obtained at all letter positions in Experinent I cannot be accounted for solely by problems of cue localization. If it could be demonstrated that a selective masking effect could be obtained with the multiple-bar-marker procedure, it would appear that this procedure, having solved the problems of cue location, can act as an aid to letter recall and. furthermore, that the results of Experiment 1 can be explained by subjects' inability to employ the single bar markers as report cues.

\section{Method}

General. The niethod was basically the same as that followed in Experiment I except that the multiple bar-marker technique developed in Experiment III was employed as the PR procedure. As in Experiment III, bar markers were placed in locations both above and below the visual-noise stimulus. For the no-masking condition. the bar markers were positioned in a similar fashion except that no visual noise stimulus was present. All other aspects of the present experiment were identical to those of Experiment $I$.

Subjects. The seven subjects in each group were selected from the same population as in Experiment 1, and they were assigned to groups in the same manner.

\section{Results and Discussion}

The data presented in Figure 3 were scored and analyzed in the same manner as in Experiment $I$.

As in Experiment $I$, the presentation of the masking stimulus signiticantly impaired recall performance, $\mathrm{F}(1,12)=5.37, \mathrm{MSe}=28.77, \mathrm{p}<.05$. Overall performance was $52 \%$ and $35 \%$ for the no-masking and masking groups, respectively. It is also evident from Figure 3 that the masking stimulus 
had a differential effect across position in the letter rows. Report of letters at the ends of the rows was unaffected by the presence of the masking stimulus, while recall of these letters from the center positions was substantially impaired. The analysis of variance revealed a significant Masking by Position interaction, $F(6,72)=4.71, \mathrm{MSe}=7.17$.

The reappearance of a selective masking effect with the use of the multiple bar-marker procedure is in accordance with the notions developed earlier. The reason that recall performance was impaired at all positions in Experiment I was because of subjects' differential ability to employ the single bar markers at the different positions of the arrays under conditions of backward masking. Thus, the present experiment establishes that when the differential ability to employ the PR cues is eliminated, selective masking effects can be obtained. Furthermore, the multiple-bar technique appears to be a vastly superior method for investigating the processing of brief visual displays, since this technique eliminates problems of cue location and, thus, it may be presumed that the recall performance achieved with this technique reflects the amount of material actually processed.

\section{EXPERIMENT $V$}

While the research to this point establishes that the typical single bar-marker technique is a rather inferior poststimulus sampling procedure, the experiments, themselves, raise additional problems which require further investigation. First, as has been noted, since Experiment II did not include a no-masking control condition, it is impossible to determine subjects' ability to employ a single bar marker under conditions of no masking. Thus, it is not possible to assess the influence of the problem of cue location on performance for the no-masking group of Experiment I. Secondly, in Experiment II, the accuracy function, for localization by position, is an "inverted-U" shape, whereas in Experiments I and IV, accuracy plots for letter recall, by position, retain the characteristic "W shape" found in other PR studies (e.g., Averbach \& Coriell, 1961; Haber \& Standing, 1969; Merikle et al., 1971). Thirdly, in Experiment II, the average accuracy of localization at the terminal positions of the arrays (Positions 1 and 7) was approximately $21 \%$, whereas for the masking condition of Experiment $I$, subjects were able to achieve approximately $45 \%$ correct recall performance at these same terminal locations.

The questions which remain, therefore, are: (a) What are the effects of cue location performance on letter recall scores when no masking stimulus is presented? (b) Why are the performance curves for the two experimental tasks so disparate in their shapes? (c) How can subjects in Experiment I achieve

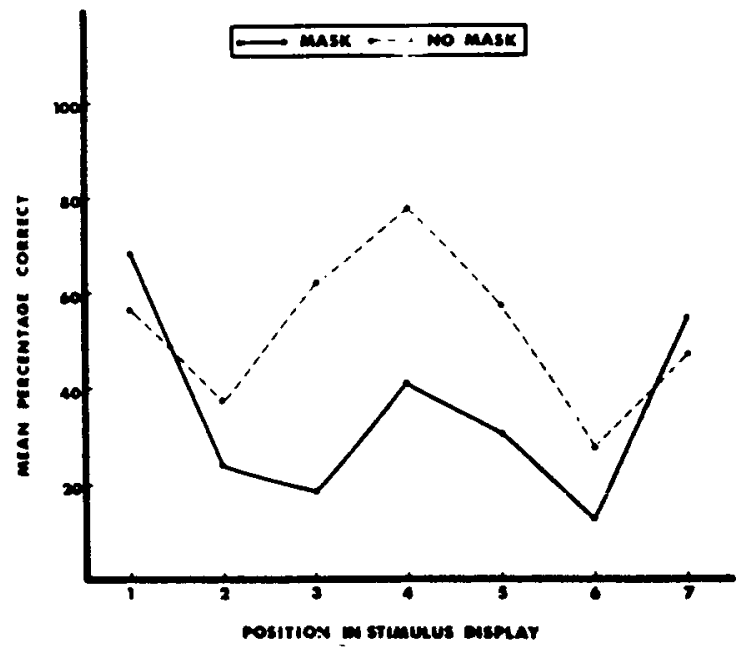

Figure 3. Mean percentage letter recall scores in Experiment IV.

recall performance scores which exceed their ability to correctly locate the probes? For, if recall performance in Experiment I was determined solely by proficiency at probe localization, then accuracy plots by position should be similar in both shape and average performance levels. At the very least (assuming that probe location is a more basic task than letter recall), it would be expected that bar location performance should set the upper limit on recall performance; recall scores should not exceed localization accuracy.

There are several possible explanations for the disparate shapes and performance levels of the accuracy plots. First, it is not unlikely that subjects in the different experiments would adopt rather different processing strategies, given the different nature of the stimulus arrays and the tasks involved. Considering the fact that subjects in the probe localization experiments (Experiments II and III) were presented with the problem of locating cues in perfectly predictable stimulus arrays, it would not be surprising to find that subjects adopted a rather different strategy from that of those subjects faced with the task of recalling single letters from unpredictable stimulus sequences (Experiments $I$ and IV). Therefore, the subjects in the different experiments may have altered their processing strategies in accordance with the nature of the stimulus arrays and/or the tasks involved and that these strategy differences would yield rather different accuracy plots and performance levels across position in the stimulus arrays.

A second possible explanation is that subjects in Experiment $I$, being uncertain about the locations of the probes (especially at the extremities of the stimulus arrays), provided their "best guess" (the subjects were instructed to guess when uncertain) when the probes were designating-the letters in the general area of the terminal locations of the arrays. This "hest guess" would not be a random choice but, 
rather, according to the "ends-tirst" processing model outlined by Merikle et al. (1971), would consist of the letters seen most clearly-the end letters. Such a strategy would cause subjects to be correct more often in their recall for the terminal locations, as these extreme letters would be reported more often in light of the uncertainty about cue position. Thus, recall performance at the ends of the letter rows would be superior to cue location accuracy. Concurrently, this "guessing strategy" "ould cause a "W-shape" accuracy plot to emerge since such a strategy would result in higher performance scores for Positions 1 and 7 than for Positions 2 and 6 , causing the performance curve to swing upward at the terminal locations of the arrays. In other words, the present account suggests that subjects' ability to recall letters from the terminal locations of the arrays may not depend upon their capacity to employ the PR cues. Rather, performance levels on these two tasks may be governed by rather different factors: cue localization may be governed by such preliminary variables as retinal acuity (which would adversely affect performance at the terminal locations), while recall performance may be determined by such "cognitive factors" as an "ends-first" processing sequence (which would aid performance at the terminal locations).

Experiment $\mathrm{V}$ was thus designed to provide some evidence which would separate the above possibilities. The rationale of the experiment was to demonstrate that subjects could perform the two tasks of cue location and letter recall concurrently, with unpredictable stimulus arrays. The procedure involved presenting rows of random letter sequences and requiring subjects, first, to report the positions designated by the probes and, secondly, to recall the letters of the arrays. In this fashion, it was expected that subjects could not adopt different processing strategies with the different tasks, since the procedure required simultaneous consideration of both probe location and letter recognition. Therefore, this procedure should eliminate such strategy differences and force subjects to adopt a single, common strategy for both tasks. Such a manipulation, therefore, should yield performance curves for both tasks which are similar in shape as well as in accuracy levels. However, if the differences in curve shapes and performance levels still remain, in spite of the attempt to force subjects to adopt a single strategy, then these differences could be attributed to the "best guess" criterion adopted by the subject in light of their uncertainty about cue position. Furthermore, such performance differences would support the suggestion that the performance on the different tasks of cue location and letter recall is determined by rather different, independent factors.

\section{Method}

Design and Materials. All subjects were presented a set of 84 sequences of seven random letters four times for a total of 336 trials. The set of sequences was constructed and prepared in the same manner as in Experiment 1 , except that each of the 21 letters enployed appeared four times in each of the seven positions. The visual angle subtended by a single sequence was approximately $4.50^{\circ} \times 0.36^{\circ}$.

On half of the trials, presentation of the letter sequences was followed by a visual noise stimulus similar to that employed in previous experiments, while on the remaining trials, a white field followed the letters. The visual noise stimulus subtended a visual angle of approximately $1.08^{\circ}$ vertically, and it was extended across the entire visual tield. All subjects completed 168 trials under one condition before proceeding to the other. Four subjects received the treatments in a masking/no-nasking sequence, while for the remaining subjects this order was reversed. In aúdition, bar-marker stimuli were prepared in the same manner as in Experiment $I$.

The 84 sequences were presented in an identical order for each subject. and the counterbalancing procedure which determined the position probed on each trial was identical to that used in Experiment 1. For each subject, the seven positions were probed an equal number of times under both masking and no-masking conditions. In addition, since each subject was presented the set of sequences four times, the counterbalancing procedure also ensured that, for cach subject, a different position of the row was probed on each presentation.

Procedure. All materials were presented by means of a Gerbrands thre-tield tachistuscope. The luminance of all fieids was approximately $6 \mathrm{tL}$. Stimulas duration conditions were identical to thuse of Experiment $I$. All subjects were instructed to report the position indicated by the bar marker and then to recall as many letters from the display as possible. in any preferred order. In reporting the positions of the probes, subjects were instructed to consider the leftmost location as the "one" position and the rightmest location as the "seven" position.

Subjects. The seven subjects were male and female undergraduate students at Trent University who were paid \$2 for their participation.

\section{Results and Discussion}

Performance scores for each subject were determined by summing separately both the number of correct localizations and the number of letters correctly recalled at each position for both masking and no-masking conditions. For each subject, a maximum score of 24 correct localizations and 168 correct identifications at each position was possible. These scores were submitted to separate 2 by 7 repeated-measures analyses of variance to evaluate the effects of masking and position. The letter recall and probe localization scores are presented in the left and right panels of Figure 4, respectively, in terms of mean percentages correct at each position for masking and no-masking conditions.

Comparing the two panels of Figure 4, the most obvious result is that the bar location and letter recall tasks resulted in quite differently shaped accuracy plots across position in the stimulus arrays. The letter recall lask resulted in the same "W-shape" curves found in Experiments $I$ and IV, while the probe location task produced the "inverted U-shape" plot found in Experiment II. 
Flgure 4. Mean percentage letter recall (left panel) and probe localization(right panel) scores in Experiment V.

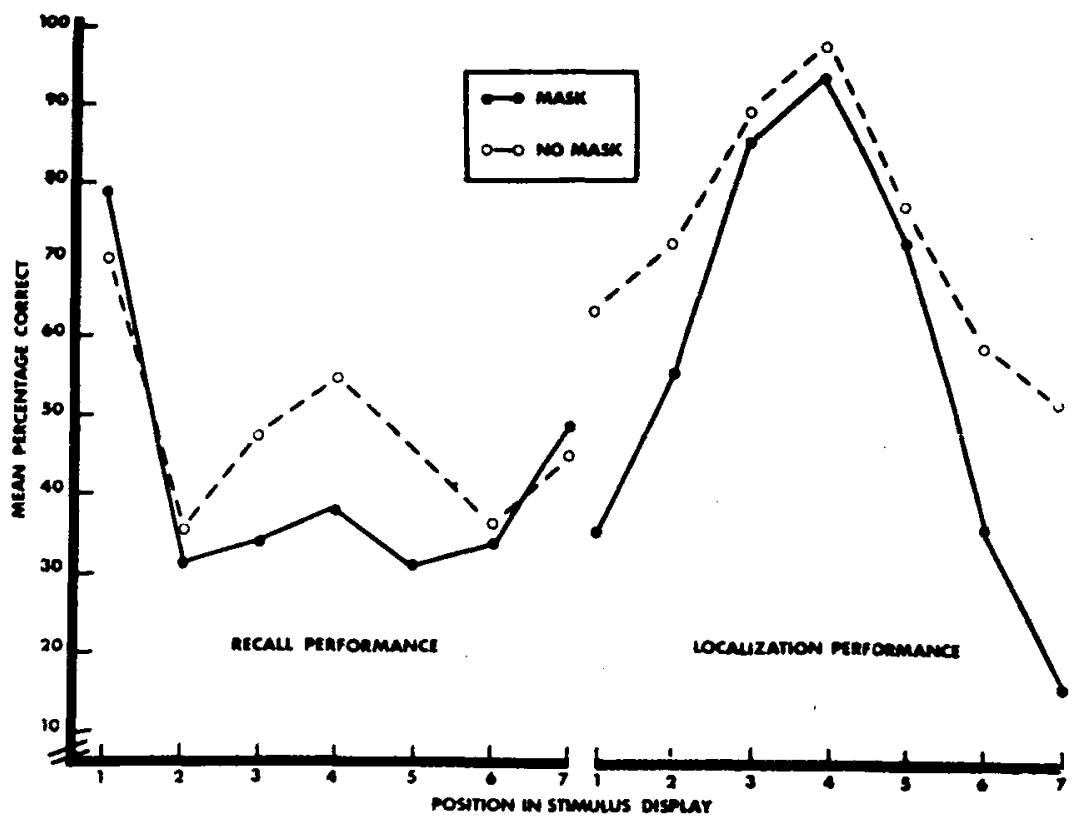

Considering letter recall performance, as portrayed in the left panel of Figure 4, it is obvious that mask presentation impaired recall performance, $F(1,6)=$ 7.24, $\mathrm{MSe}=369.2, \mathrm{p}<.05$, Overall recall pertormance was $48 \%$ and $42 \%$ for the no-masking and masking conditions, respectively. Furthermore, as in Experiment IV, the largest effects of masking were at the center positions of the letter rows, while report of the letters of the ends of the arrays was unaffected by mask presentation. This differential effect of the masking stimulus is supported by a significant Masking by Position interaction, $F(6,36$, $=11.93, \mathrm{MSe}=125.3$.

An inspection of the right panel of Figure 4 indicates that mask presentation also impaired probe location performance, $F(1,6)=49.1, \mathrm{MSe}=7.33$. Overall, location performance was $74 \%$ and $56 \%$ for the no-masking and masking conditions, respectively. As in Experiment II, performance decreased directly with the distance of the position probed from fixation for both masking and no-masking conditions, $F(6,36)$ $=10.8, \mathrm{MSe}=32.61$, and a nonsignificant Mask by Position interaction, $\mathrm{F}(6,36)=1.52, \mathrm{MSe}=22.4$, indicated that the location performance at the different positions was not differentially intluenced by mask presentation.

Lastly, a comparison of performance levels for the two tasks at the terminal positions of the arrays indicates that letter recall performance exceeded probe localization scores, especially under masking conditions. For the masking condition, cue location performance was $36 \%$ and $15 \%$ at Positions 1 and 7 , respectively, while letter recall performance was $79 \%$ and $48 \%$, respectively. For the no-masking condition, cue location scores were $63 \%$ and $54 \%$ at Positions 1 and 7 , respectively, while letter recall scores were $70 \%$ and $45 \%$, respectively. Friedman two-way analysis of variance performed upon percentage correct scores of the masking data for the two tasks revealed that, at both Positions 1 and 7, letter recall performance was superior to cue location performance, $\chi^{2} r(1)=7.0$, $p<.01$ (in both cases). Similar analysis of the no-masking data indicated that letter recall and cue location scores were not significantly different.

The implications of the present experiment are clear. It is evident that the requirement to concurrently locate the probe and recall the letters does not result in similar shapes in the accuracy plots for the two tasks. Thus, it appears that quite different factors determine the shapes of the curves. Probe location performance is determined by retinal acuity, while letter recall scores are governed by the order of processing. Furthermore, the presence of the selective masking effect at the different positions of the rows for letter recall indicates that processing of the stimulus arrays was not affected by the probe location task. Thus, it seems that subjects carry out the tasks of probe location and letter recall independently, maintaining the "ends-first" processing sequence (Merikle et al., 1971) in spite of the requirements to locate the probe.

In addition, the probe location scores indicate that cue location is problematic for subjects regardless of whether a masking stimulus is presented. Thus it appears that these difficulties would affect letter recall performance under both masking and no-masking conditions when bar markers are employed as $P R$ cues as in Experiment 1.

Lastly, the present results support the notion that subjects are providing their "best guess" for the 
letters cued at the extremes of the arrays. Since letter recall scores exceeded cue location performance at the extrene locations for the masking condition and recall performance at the ends of the rows was not impaired by mask presentation, it is obvious that subjects have successfully processed and are able to report much more from the stimulus arrays than performance based upon the bar-marker technique reveals. In short, the typical bar-marker PR procedure is a rather inferior technique and, subsequently, inferences made about the processing of brief stimulus arrays may be erroneous if they are based upon the performance levels achieved with this PR technique.

\section{REFERENCES}

Averbach, E., \& Coriell, A. S. Short-term memory in vision. Bell Sistem Technical Journal, 1961, 40, 309-328.

Coltheart, $M$. Visual intormation processing. In $P$. C. Dodwell (Ed.). New horizons in psychology 2. Harmondsworth: Penguin, 1972.

GREGORY, R. L. Eye and bruin: The psychology of seeing. Toronto: McGraw-Hill, 1966.
HABER. R. N. Information processing analyses of visual perception: An introduction. In R. N. Haber (Ed.), Information processing approaches to visual perception. New York: Holt. Rinehart, \& Winston, 1969. Pp. 1-15.

Haber, R. N., \& Standing, L. Location of errors with a post-stimuli,s indicator. Psychonomic Science, 1969, 17. 345-346.

Neisser, U. Cognitive psychology. New York: Appleton-CenturyCrofts, 1967.

Merikle, P. M. \& Coltheart, M. Selective forward masking. Canadian Journal of Psychology. 1972, 26. 296-302.

Merikle, P. M., Coltheart, M., \& Lowe, D. G. On the selective effects of a patterned masking stimulus. Canadian Journal of Psychology. 1971, 25, 264-279.

TownSEND, V. M. Loss of spatial and identity information following a tachistoscopic exposure. Jourtal of Experimental Psychology. 1973, 98, 113-118.

\section{NOTE}

1. The rejection region fir all statistical tests reported in this paper is $p<.005$, unless otherwise stated.

(Received tor publication April 30, 1975; accepled June 16, 1975.) 